\title{
GASEIFICAÇÃO DE BIOCHAR OBTIDO DA PIRÓLISE DE BAGAÇO DE MAÇÃ VISANDO SUA UTILIZAÇÃO PARA GERAÇÃO DE ENERGIA
}

\author{
T. R. PACIONI ${ }^{1}$, R. F. P. M. MOREIRA ${ }^{1}$ e H. J. JOSÉ ${ }^{1}$ \\ ${ }^{1}$ Universidade Federal de Santa Catarina, Departamento de Engenharia Química e Engenharia de \\ Alimentos \\ E-mail para contato: tatiana.pacioni@gmail.com
}

\begin{abstract}
RESUMO - A conversão termoquímica de biomassa representa uma alternativa viável de geração de energia, atuando ao mesmo tempo como solução para a disposição final de rejeitos. Neste trabalho foi estudada a capacidade de gaseificação do biochar de bagaço de maçã. Inicialmente, a biomassa bruta foi pirolisada em duas condições diferentes: pirólise rápida e lenta. Embora a pirólise lenta tenha apresentado maior rendimento de biochar, as proporções de carbono fixo e matéria volátil dos dois biochars ficaram muito próximas. As respectivas reatividades foram avaliadas com $\mathrm{CO}_{2} \mathrm{em}$ um analisador termogravimétrico e os parâmetros cinéticos calculados utilizando-se o modelo cinético que melhor se ajustou aos dados experimentais. O biochar obtido da pirólise rápida apresentou maior reatividade, especialmente para temperaturas mais elevadas. Os resultados do estudo cinético confirmaram, para os dois tipos de pirólise avaliados, o potencial de aproveitamento energético do bagaço de maçã por meio do processo de gaseificação.
\end{abstract}

\section{INTRODUÇÃO}

A demanda energética mundial tem crescido de forma constante e acelerada. De acordo com o relatório Perspectivas Energéticas Internacionais 2013, publicado pelo Departamento de Energia dos EUA (U.S. EIA, 2013), o consumo de energia entre 2010 e 2040 aumentará em 56 \%, sendo a maior proporção referente aos países não pertencentes à OECD. Atualmente os combustíveis fósseis são as principais fontes de energia, e o relatório do EIA estima que até 2040 eles ainda serão responsáveis pelo suprimento de $80 \%$ da demanda de energia global. Ao mesmo tempo, a intensa exploração de combustíveis desta categoria apresenta alguns desafios, como a previsão de esgotamento das reservas, questões financeiras, questões ambientais, e conflitos militares e geopolíticos (Surisetty et al., 2011; García et al., 2012). A diversificação da matriz energética pode se mostrar como uma forma sustentável de suprir as demandas no futuro, sem comprometer os recursos existentes. Por isso, é importante se desenvolver pesquisas que visem o aprimoramento de materiais alternativos para obtenção de energia. Dentre as opções disponíveis, os combustíveis renováveis têm recebido destaque nos últimos anos, especialmente os chamados biocombustíveis (incluindo biomassa, resíduos sólidos municipais e resíduos industriais e agro-industriais), devido a vantagens como baixo potencial poluidor, ampla distribuição, neutralidade em relação à emissão de $\mathrm{CO}_{2}$ (Yuan et al., 2011). 


\section{9 a 22 de outubro de 2014 \\ Florianópolis/SC}

Os resíduos sólidos representam uma parcela importante na classe de biocombustíveis, uma vez que a sua geração aumenta a cada ano e a gestão destes materiais se torna um problema. Segundo dados do Ministério do Meio Ambiente (2011), as intensas atividades industriais e agroindustriais brasileiras geram grandes quantidades de resíduos - 97,7 e 290,8 mil ton/ano, respectivamente. A conversão termoquímica é uma das formas de aproveitamento desses resíduos, em evidência recentemente por sua versatilidade tanto de utilização de matéria prima quanto em relação aos produtos gerados. Neste contexto, a gaseificação de resíduos agroindustriais se apresenta como uma das alternativas mais convenientes para a produção de energia, porque além de ser um processo eficiente e limpo, também atua como uma forma adequada de descarte dos mesmos.

O objetivo deste estudo é avaliar o efeito das condições de pirólise na reatividade do biochar de bagaço de maçã, além de estudar a sua capacidade de gaseificação $\operatorname{com} \mathrm{CO}_{2}$, tomando como base modelos cinéticos empíricos e semi-empíricos.

\section{MATERIAIS E MÉTODOS}

\subsection{Amostra}

A biomassa utilizada neste estudo foi o bagaço de maçã, gerado como resíduo na produção do suco de maçã industrial. O componente majoritário desta biomassa é a matéria volátil (MV), com $80,55 \%$, seguido do carbono fixo (CF), com 17,66 \% e das cinzas (CZ), com 1,79\%. Nas cinzas, os componentes inorgânicos em maior quantidade são potássio $(31,27 \%)$, fósforo $(18,52 \%)$ e cálcio $(13,44 \%)$.

\subsection{Pirólise}

Os experimentos de pirólise foram realizados em um reator de quartzo tubular de leito fixo, inserido em um forno elétrico acoplado a um controlador de temperatura digital. Antes de cada experimento foi realizada uma purga com $\mathrm{N}_{2}$ durante 15 minutos em temperatura ambiente. $\mathrm{Na}$ pirólise lenta a amostra foi aquecida até $600{ }^{\circ} \mathrm{C}$ com uma taxa de aquecimento média de $12{ }^{\circ} \mathrm{C} / \mathrm{min} \mathrm{e}$ tempo de residência de 60 minutos. Na pirólise rápida a amostra foi aquecida até $900{ }^{\circ} \mathrm{C}$, com uma taxa de aquecimento média de $230{ }^{\circ} \mathrm{C}$ e tempo de residência de 15 minutos. Por fim, a amostra era resfriada até a temperatura ambiente, mantendo a atmosfera de $\mathrm{N}_{2}$. O rendimento do biochar foi determinado pela perda de massa total durante a pirólise.

\subsection{Gaseificação}

Os experimentos de gaseificação com $\mathrm{CO}_{2}$ foram realizados em um analisador termogravimétrico (Shimadzu, DTG-60/60H), em pressão atmosférica. A partir de testes variando o tamanho da partícula, o fluxo de $\mathrm{CO}_{2}$ e a quantidade de amostra, definiu-se que as condições nas quais o regime era controlado pela reação química foram: tamanho de partícula menor do que $106 \mu \mathrm{m}$, fluxo de $\mathrm{CO}_{2}$ de $200 \mathrm{ml} / \mathrm{min}$ e massa de amostra aproximadamente $12 \mathrm{mg}$. O procedimento experimental da gaseificação era iniciado com a purga com $\mathrm{N}_{2}$ por 60 minutos, em temperatura ambiente. Depois desse período era iniciado o aquecimento até $900{ }^{\circ} \mathrm{C}$, a uma taxa constante de 
$10{ }^{\circ} \mathrm{C} / \mathrm{min}$. Em seguida, a temperatura era reduzida para cada temperatura de reação $(760,810$ e $860^{\circ} \mathrm{C}$ ) e, depois de atingida essa temperatura, o gás era trocado para $\mathrm{CO}_{2}$ para iniciar a gaseificação isotérmica. A reação era procedida até que não fosse observada variação na massa. Para cada temperatura de reação foi realizado um experimento em branco, nas mesmas condições descritas acima, porém sem amostra no cadinho.

A conversão da reação $(\mathrm{X})$ e a reatividade instantânea $(\mathrm{r})$ foram calculadas utilizando as seguintes equações:

$$
\begin{gathered}
X=\frac{m_{0}-m}{m_{0}-m_{C Z}} \\
r=-\frac{1}{m_{0}} \cdot \frac{d m}{d t}=\frac{d X}{d t}
\end{gathered}
$$

onde $m_{0}$ é a massa no início da gaseificação, $m$ é a massa instantânea no tempo t e $m_{C Z}$ é a massa de cinzas restante no final da gaseificação.

\subsection{Modelos Cinéticos}

Se os efeitos de transferência de massa interna e externa podem ser desprezados, a taxa global da reação gás-sólido é totalmente controlada pela reação química na superfície do sólido. Uma expressão geral para a taxa da gaseificação char $-\mathrm{CO}_{2}$, com concentração constante de $\mathrm{CO}_{2}$, pode ser representada pela Equação 3.

$$
\frac{d X}{d t}=k(T) \cdot f(X)
$$

onde $k(T)$ é a constante da taxa e $f(X)$ é uma função que representa a mudança estrutural no biochar durante as reações de gaseificação.

Diferentes modelos cinéticos têm sido propostos para representar a reatividade do biochar a partir da função $f(X)$. Os modelos teóricos foram desenvolvidos originalmente para descrever a gaseificação de carvão, porém também são aplicados para a gaseificação de biomassas. Os três modelos mais comuns para descrever a cinética da reação gás-sólido são: o Modelo Homogêneo (MH), o Modelo do Núcleo Não Reagido (MNNR) e o Modelo de Poros Aleatórios (MPA). Zhang et al. (2008) sugeriram uma modificação no Modelo de Poros Aleatórios introduzindo dois parâmetros adimensionais, $c$ e $p$, com o objetivo de descrever sistemas que apresentam reatividades máximas para altas conversões, casos onde o MPA não é aplicável.

Para os modelos teóricos foi aplicado o método integral de análise para o cálculo dos parâmetros cinéticos, utilizando os dados experimentais na faixa de conversão entre 5 e $80 \%$. $\mathrm{Na}$ Tabela 1 são apresentadas as equações da taxa de cada modelo e as respectivas equações linearizadas. $\mathrm{Na}$ equação do MPA, $\psi$ é um parâmetro relacionado com a estrutura porosa inicial do biochar. Neste trabalho, este parâmetro foi calculado pelo método da unificação (Lahijani et al., 2012). Para o Modelo de Poros Aleatórios Modificado (MPAM) foi utilizado o método diferencial de análise, e os parâmetros foram obtidos com a equação apresentada na Tabela 1, a partir do método dos mínimos 
quadrados. O cálculo da conversão foi realizado com o método numérico de Runge Kutta de ordem 4, implementado no software Excel.

Tabela 1 - Equações do Modelo Homogêneo, Modelo do Núcleo Não Reagido, Modelo de Poros Randômicos e Modelo de Poros Aleatórios Modificado.

\begin{tabular}{ccc}
\hline Modelo & Equação da taxa & Forma linearizada \\
\hline MH & $\frac{d X}{d t}=k_{M H} \cdot(1-X)$ & $-\ln (1-X)=k_{M H} \cdot t$ \\
MNNR & $\frac{d X}{d t}=k_{M N N R} \cdot(1-X)^{2 / 3}$ & $3\left[1-(1-X)^{1 / 3}\right]=k_{M N N R} \cdot t$ \\
MPA & $\frac{d X}{d t}=k_{M P A} \cdot(1-X) \cdot \sqrt{1-\psi \cdot \ln (1-X)}$ & $\left(\frac{2}{\psi}\right) \cdot[\sqrt{1-\psi \cdot \ln (1-X)}-1]=k_{M P A} \cdot t$ \\
MPAM & $\frac{d X}{d t}=k_{M P A M} \cdot(1-X) \cdot \sqrt{1-\psi \cdot \ln (1-X)} \cdot\left(1+(c \cdot X)^{p}\right)$ & - \\
\hline
\end{tabular}

A constante da taxa, $k_{i}$, ajustada para cada modelo $\left(k_{M H}, k_{M N N R}, k_{M P A}\right.$ e $\left.k_{M P A M}\right)$ nas três temperaturas pode ser expressa pela equação de Arrhenius, da seguinte forma:

$$
k_{i}=k_{0} \cdot \exp \left(-\frac{E_{A}}{R \cdot T}\right)
$$

onde $k_{0}, E_{A}, R$ e $T$ são o fator pré-exponencial, a energia de ativação, a constante dos gases e a temperatura, respectivamente. Conhecer os valores de $E_{A}$ e $k_{0}$ de uma reação é muito importante para diversas aplicações, como a interpretação dos mecanismos da reação, do fenômeno catalítico e do comportamento molecular, o desenvolvimento de projetos e a otimização dos parâmetros de gaseificadores e também para a modelagem e a simulação dos reatores (Nowicki et al., 2011).

\section{RESULTADOS E DISCUSSÃO}

\subsection{Resultados da pirólise}

A Tabela 2 apresenta os resultados de análise imediata e os rendimentos de biochar para as duas condições de pirólise estudadas.

Tabela 2 - Propriedades dos biochars de Bagaço de Maçã da pirólise lenta e rápida

\begin{tabular}{ccc}
\hline & Biochar - Pirólise lenta & Biochar - Pirólise rápida \\
\hline Análise imediata & & \\
\hline MV (\%, b.s.) & 14,24 & 10,28 \\
CF (\%, b.s.) & 78,42 & 80,62 \\
CZ (\%, b.s.) & 7,34 & 9,11 \\
\hline Rendimento & & \\
\hline Rendimento da pirólise (\%) & 23,2 & 17,7 \\
\hline
\end{tabular}

É possível observar que as proporções dos elementos da análise imediata ficaram muito próximas, embora o rendimento da pirólise lenta tenha sido superior. Na pirólise lenta, o rendimento é maior porque a matéria volátil permanece mais tempo em contato com o resíduo carbonoso, favorecendo as reações de recombinação dos fragmentos de alcatrão. 


\section{9 a 22 de outubro de 2014 \\ Florianópolis/SC}

\subsection{Reatividade}

As Figuras 1a e 1b apresentam os perfis de reatividade para o biochar de Bagaço Maçã da pirólise lenta e rápida, respectivamente.
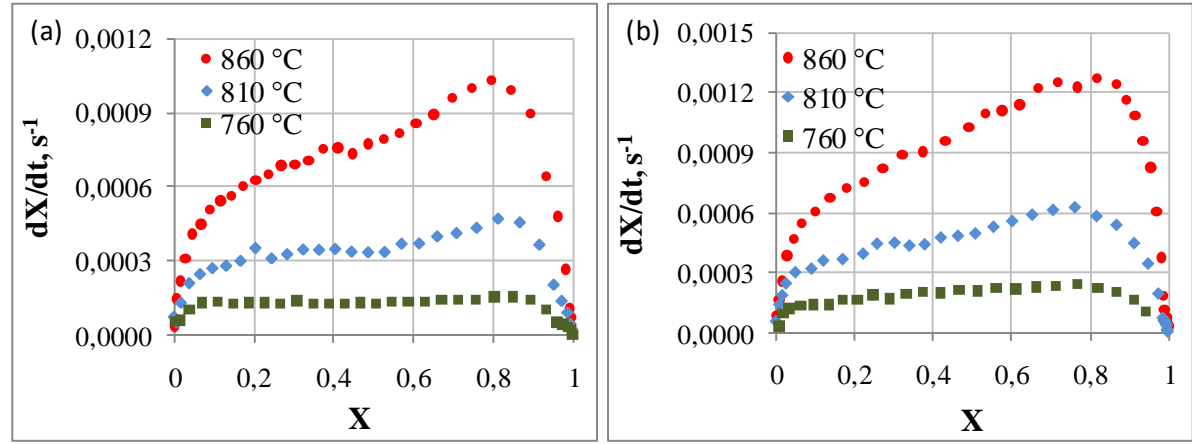

Figura 1 - Perfil de reatividade para o biochar do Bagaço de Maçã obtido (a) na pirólise lenta e (b) na pirólise rápida

Observando os gráficos acima é possível analisar o efeito causado pela diferença na história térmica do biochar. Os valores da taxa durante a reação foram mais elevados para o biochar da pirólise rápida, apesar de o perfil das curvas terem se mantido iguais. Como descrito anteriormente, as amostras de biomassa foram submetidas, em cada pirólise, a diferentes taxas de aquecimento, temperaturas e tempos de residência. Cada um destes fatores é reportado na literatura como exercendo uma influência individual na taxa da reação: o aumento da taxa de aquecimento aumenta a reatividade, enquanto que temperaturas e tempos de residência maiores contribuem para a sua diminuição. $\mathrm{O}$ efeito sinérgico das variações testadas resultou no aumento da reatividade do biochar da pirólise rápida nas três temperaturas, ou seja, a influência positiva do aumento da taxa de aquecimento e da diminuição do tempo de residência foi mais expressiva do que a influência negativa do aumento na temperatura. Este resultado foi obtido porque as reações de recombinação do resíduo carbonoso, favorecidas na pirólise lenta devido ao maior tempo de contato do biochar com a matéria volátil, diminuem os sítios ativos disponíveis.

\subsection{Estudo Cinético}

Para a análise dos dados da gaseificação do Bagaço de Maçã pelo método isotérmico, primeiramente o modelo homogêneo, núcleo não reagido e poros aleatórios foram ajustados aos dados experimentais utilizando as equações da Tabela 1. Os resultados dos ajustes em termos de conversão para cada modelo são apresentados nas Figuras 2, 3 e 4 (a linha contínua representa o ajuste dos dados experimentais com o modelo).

Como é possível observar com as curvas de conversão em função do tempo experimentais e ajustadas, o melhor modelo teórico foi o Modelo de Poros Aleatórios. Porém, este modelo apresentou desvios para conversões acima de 0,7 . Isso ocorre porque o modelo não é capaz de descrever os picos em alta conversão das curvas de reatividade apresentadas nas Figuras 1a e 1b. Estes picos são atribuídos à ação catalítica da matéria mineral presente na biomassa (Suzuki et al., 2011). 

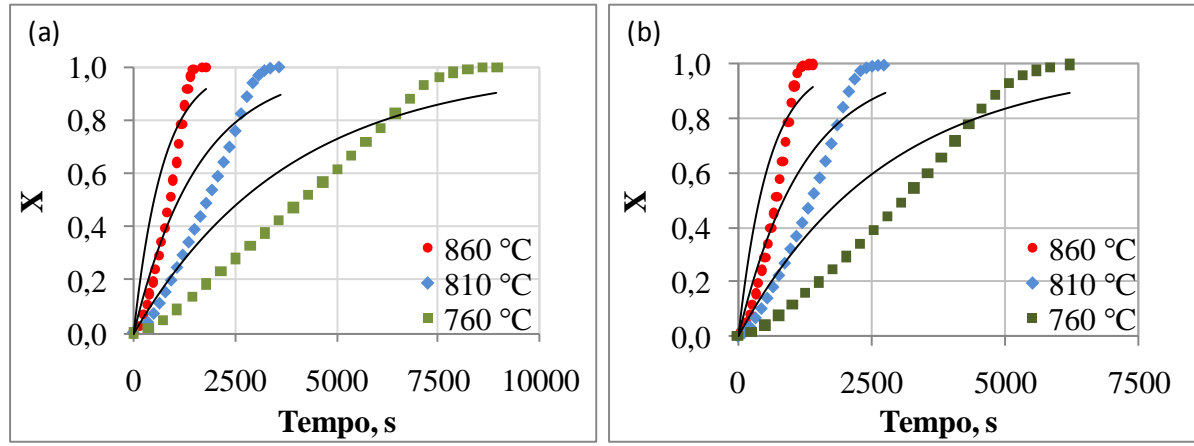

Figura 2 - Dados de gaseificação do Bagaço de Maçã pelo modelo homogêneo para (a) o biochar da Pirólise Lenta e (b) o biochar da Pirólise Rápida
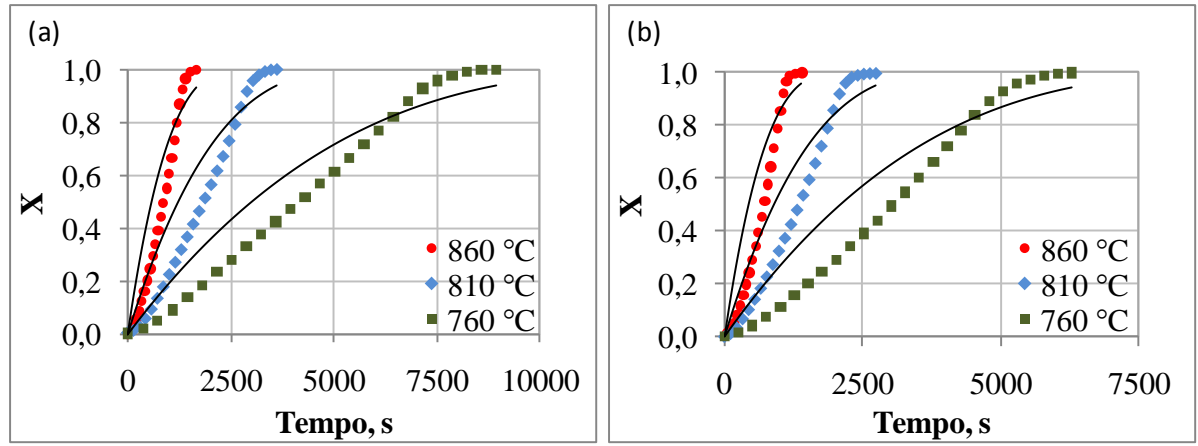

Figura 3 - Dados de gaseificação do Bagaço de Maçã pelo modelo do núcleo não reagido para (a) o biochar da Pirólise Lenta e (b) o biochar da Pirólise Rápida
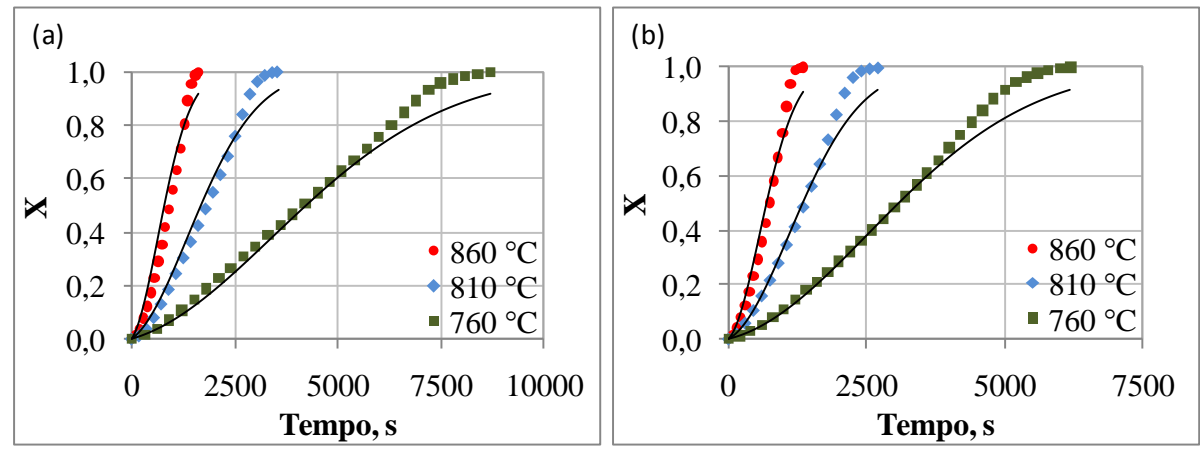

Figura 4 - Dados de gaseificação do Bagaço de Maçã pelo modelo de poros aleatórios para (a) o biochar da Pirólise Lenta e (b) o biochar da Pirólise Rápida

Por conta da falta de ajuste observada para os modelos teóricos, o modelo semiempírico proposto por Zhang et al. (2008) também foi testado. Os resultados do ajuste em termos de conversão para o Modelo de Poros Aleatórios Modificado são apresentados na Figura 5. 

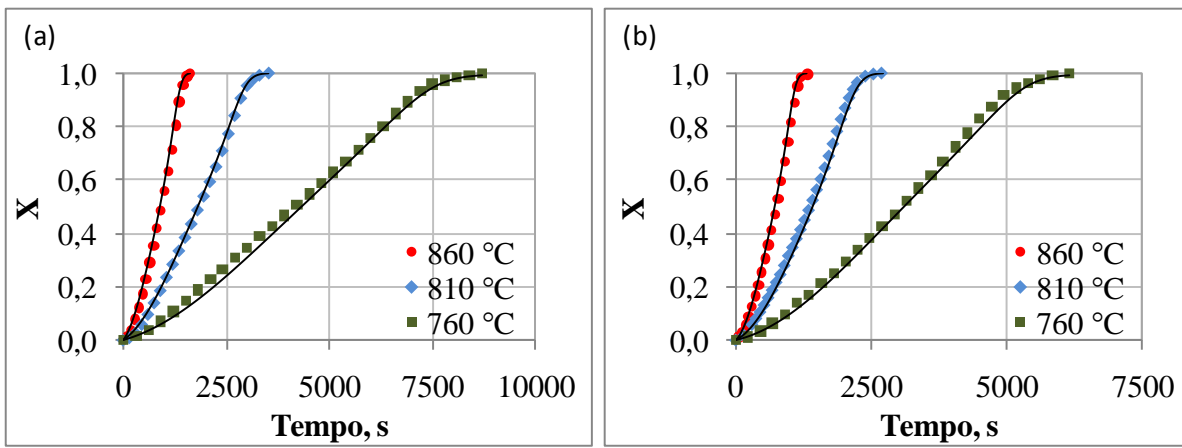

Figura 5 - Dados de gaseificação do Bagaço de Maçã pelo modelo de poros aleatórios modificado para (a) o biochar da Pirólise Lenta e (b) o biochar da Pirólise Rápida

As Tabelas 3 e 4 apresentam a comparação dos parâmetros cinéticos calculados para o biochar de BM obtido na pirólise lenta e rápida, respectivamente, pelos quatro modelos utilizados, junto com o coeficiente de correlação não linear para o ajuste dos dados experimentais.

Tabela 3 - Comparação dos parâmetros cinéticos determinados para o biochar de Bagaço de Maçã da Pirólise Lenta.

\begin{tabular}{lcccc}
\hline Parâmetro & Homogêneo & Não reagido & Poros Aleatórios & $\begin{array}{c}\text { Poros Aleatórios } \\
\text { Modificado }\end{array}$ \\
\hline $\mathrm{E}_{\mathrm{a}}(\mathrm{kJ} / \mathrm{mol})$ & 165,8 & 167,1 & 170,5 & 161,5 \\
$\mathrm{k}_{\mathrm{o}}\left(\mathrm{s}^{-1}\right)$ & $6,70 \cdot 10^{4}$ & $4,33 \cdot 10^{4}$ & $2,00 \cdot 10^{4}$ & $7,33 \cdot 10^{3}$ \\
$\Psi$ & --- & --- & 51,8 & 51,8 \\
$\mathrm{c}$ & --- & --- & --- & 1,30 \\
$\mathrm{p}$ & --- & --- & --- & 4,96 \\
$\mathrm{R}^{2}$ & 0,765 & 0,850 & 0,972 & 0,999 \\
\hline
\end{tabular}

Tabela 4 - Comparação dos parâmetros cinéticos determinados para o biochar de Bagaço de Maçã da Pirólise Rápida.

\begin{tabular}{|c|c|c|c|c|}
\hline Parâmetro & Homogêneo & Não reagido & Poros Aleatórios & $\begin{array}{c}\text { Poros Aleatórios } \\
\text { Modificado }\end{array}$ \\
\hline $\mathrm{E}_{\mathrm{a}}(\mathrm{kJ} / \mathrm{mol})$ & 154,8 & 154,8 & 153,8 & 147,1 \\
\hline $\mathrm{k}_{\mathrm{o}}\left(\mathrm{s}^{-1}\right)$ & $1,98 \cdot 10^{4}$ & $1,60 \cdot 10^{4}$ & $3,00 \cdot 10^{3}$ & $1,30 \cdot 10^{4}$ \\
\hline$\Psi$ & --- & --- & 101,0 & 101,0 \\
\hline $\mathrm{c}$ & --- & --- & --- & 1,30 \\
\hline $\mathrm{p}$ & --- & --- & --- & 4,98 \\
\hline $\mathrm{R}^{2}$ & 0,781 & 0,829 & 0,970 & 0,997 \\
\hline
\end{tabular}

Os resultados das Tabelas 3 e 4 indicam uma diminuição na energia de ativação da amostra de bagaço de maçã que foi pirolisada com taxa de aquecimento alta, em relação à amostra da pirólise lenta, confirmando as discussões do item anterior. Os coeficientes de correlação permitem verificar que o modelo de poros aleatórios modificado se ajustou melhor aos dados experimentais $\left(\mathrm{R}^{2}>0,99\right)$. Os valores elevados de $\psi$ representam uma estrutura pouco uniforme com muitas mudanças na estrutura dos poros durante a progressão da reação. Os valores obtidos das constantes $c$ e $p$, para o MPAM das duas amostras, estão coerentes com os resultados apresentados por Zhang et al. (2008), quando eles relacionaram estes parâmetros com a quantidade de potássio na amostra. Segundo estes 


\section{9 a 22 de outubro de 2014 \\ Florianópolis/SC}

autores, quanto maior o teor de potássio na amostra, menor o valor de $p$ e maior o valor de $c$. Conforme mostrado nas Tabelas 2 e 3, os biochars de bagaço de maçã apresentaram valores muito próximos, o que sugere que o metal não foi volatilizado nas duas condições de pirólise aplicadas à biomassa.

\section{CONCLUSÕES}

Com os resultados do trabalho foi possível observar que, embora a pirólise lenta tenha apresentado maior rendimento de biochar, as proporções da análise imediata dos dois biochars se mantiveram as mesmas. Avaliando os resultados da gaseificação dos biochars, o melhor desempenho foi alcançado para o produto da pirólise rápida, porque, nas condições estudadas, o efeito positivo do aumento da taxa de aquecimento e da diminuição do tempo de residência se sobrepôs ao efeito negativo da temperatura mais elevada. O modelo melhor ajustado foi o Modelo de Poros Aleatórios Modificado, que leva em conta a influência catalítica do potássio na reação. Os parâmetros cinéticos encontrados ficaram próximos ao que é encontrado na literatura, confirmando o potencial de aproveitamento energético do bagaço de maçã por meio do processo de gaseificação.

\section{REFERENCIAS}

ABRELPE - Associação Brasileira de Empresas de Limpeza Pública e Resíduos Especiais. Panorama dos Resíduos Sólidos no Brasil. 2012.

GARCÍA, R.; PIZARRO, C.; LAVÍN, A. G.; BUENO, J. L. Characterization of spanish biomass wastes for energy use. Bioresource Technol, v. 103, n. 1, p. 249-258, 2012.

LAHIJANI, P.; ZAINAL, Z. A.; MOHAMED, A. R.; MOHAMMADI, M. Ash palm empty fruit bunch as a natural catalyst for promoting the $\mathrm{CO}_{2}$ gasification reactivity of biomass char. Bioresource Technol, v. 132, p. 351-55, 2012.

MMA - Ministério do Meio Ambiente. Plano Nacional de Resíduos Sólidos. Versão Preliminar para Consulta Pública. Brasília, setembro, 2011.

NOWICKI, L.; ANTECKA, A.; BEDYK, T.; STOLAREK, P.; LEDAKOWICZ, S. The Kinetics of gasification of char derived from sewage sludge. J Therm Anal Calorim, v. 104, n. 2, p. 693-700, 2011.

SURISETTY, V. R.; KOZINSKI, J.; DALAI, A. K. Biomass, availability in Canada, and gasification: an overview. Biomass Conv Bioref, v. 2, n. 1, p. 73-85, 2011

SUZUKI, T.; NAKAJIMA, H.; IKENAGA, N.; ODA, H.; MIYAKE, T. Effect of mineral matters in biomass on the gasification rate of their chars. Biomass Conv Bioref, v. 1, n. 1, p. 17-28, 2011

U.S. EIA, 2013, http://www.eia.gov/forecasts/ieo/

YUAN, S.; CHEN, X.; LI, J.; WANG, F. $\mathrm{CO}_{2}$ gasification kinetics of biomass char derived from hightemperature rapid pyrolysis. Energ Fuel, v. 25, n. 5, p. 2314-21, 2011.

ZHANG, Y.; ASHIZAWA, M.; KAJITANI, S.; MIURA, K. Proposal of a semi-empirical kinetic model to reconcile with gasification reactivity profiles of biomass chars. Fuel. v. 87, n. 4-5, p. 475481, 2008. 